\title{
Liver biopsy under ultrasound control: implications for training in the Calman era
}

Liver biopsy is the standard procedure for the investigation of a wide range of liver pathologies. It is generally a safe procedure but mortality rates range from 1:1000 to $1: 10000 .{ }^{12}$ Although a biopsy sample is traditionally taken "blind", many centres now carry out biopsies under ultrasound control, and this change in technique has impacted directly on the training of general physicians and gastroenterologists. In this article, we review the evidence for the safety of a real time ultrasound guided approach against the traditional blind biopsy, and examine the implications of this change for trainee clinicians who rotate through centres who now use an ultrasound guided technique.

The basic technique for liver biopsy was first described by Sherlock in $1945^{3}$; the earliest approaches used Tru-Cut and Menghini needles to remove tissue and required inpatient care for 24 hours after the procedure. More recently, liver biopsy patients have been day cases, with no evidence of increased risk. ${ }^{4}$ Histological diagnosis provides useful guidance for the future management of many liver conditions, and is particularly useful in the recommendation of interferon treatment for patients with hepatitis C. The wide availability of ultrasonography has increased the use of direct visualisation in liver biopsy which has, consequently, improved the diagnostic rate in patients with focal lesions. ${ }^{5}$ However, the benefit of an ultrasound guided biopsy for diffuse or generalised disease is less clear, although some, ${ }^{6}$ but not all, ${ }^{7}$ studies report additional benefits when using ultrasonography.

Morbidity and mortality following liver biopsy are generally due to uncontrolled bleeding from inadvertent capsular tear or biopsy of intrahepatic or portal vessels. Furthermore, biliary peritonitis, due to biopsy of the gall bladder, also has a high mortality rate. Theoretically, the continuous visualisation ultrasound guided approach should reduce the rate of perforation of gall bladder and intrahepatic vessels; however, evidence to support reduced morbidity and mortality is weak. To our knowledge, no studies have shown a reduction in mortality rates between biopsies performed traditionally and biopsies guided by ultrasonography. Furthermore, such comparative studies are often compromised by the small numbers of patients involved, making it difficult to detect any differences in mortality. However, in a prospective, randomised trial, Lindor et al compared the two techniques and showed significantly lower morbidity (pain, hypotension, bleeding, and rate of subsequent hospitalisation) when using the ultrasound guided approach. ${ }^{6}$ Thus, many centres ${ }^{8}$ have started to perform ultrasound guided biopsies exclusively. This change in methodology has major implications for registrars in general medicine and gastroenterology-for example, trainees involved in the South Trent registrar rotation, who move through six hospitals, four of which now use an ultrasound guided technique.

The Joint Committee on Higher Medical Training (JCHMT) general professional training requirements of the Royal College of Physicians state that liver biopsy is classified as an "additional requirement" and that "trainees must have a sound working knowledge but need not have personally experienced this situation". ${ }^{9}$ In contrast, the
JCHMT and European accreditation requirements for trainees in gastroenterology specify a minimum of 50 procedures performed using either a blind or ultrasound guided approach and state that "trainees should be familiar with the indications, methods and risks of percutaneous liver biopsy" including those performed under ultrasound control. However, they advise that "supervised experience of a minimum of 300 abdominal ultrasound examinations" is required by trainees, in line with European accreditation recommendations. ${ }^{10}$ Linkage of experience in abdominal ultrasonography with experience in liver biopsy (as opposed to diagnosis of abdominal pathology) is not specified. Training requirements of the American Gastroenterology Board, for trainees who have a special interest in hepatology, are a minimum of 20 liver biopsies and experience in ultrasound marking for percutaneous liver biopsy (the $\mathrm{X}$ marks the spot technique). ${ }^{11}$

Therefore, present guidelines from the various bodies do not tackle the fundamental problem of a shift to real time ultrasonography in performing liver biopsy and the much greater imaging experience that it requires. Training regulations for specialist registrars in radiology specify that in year one, "they should have performed ultrasonography under the supervision of a recognised instructor", and that during years two and three they should "obtain practical experience in abdominal ultrasound" and ultimately they should have "familiarity with the relevant application of...ultrasound guided biopsy". ${ }^{12}$ In our hospital, these recommendations mean that trainees in radiology are not allowed to perform this procedure unsupervised until they have had about three years' experience (at least 500 examinations).

Is it appropriate to assume that gastroenterologists do not need to go through such a rigorous training scheme because they are working only on the liver? Even in hospitals which advocate "X marks the spot" training for gastroenterologists, the minimum requirement is that an operator is proficient in the diagnosis of dilated intrahepatic ducts and the presence of ascites, as biopsy of patients with these conditions has a much higher risk of morbidity and mortality. As a result, trainees in gastroenterology in our hospital (which uses the ultrasound guided approach exclusively) are only allowed to perform this procedure when they are proficient in full abdominal (but not pelvic) ultrasound; this has resulted in their virtual exclusion from performing liver biopsies and, since the introduction of our exclusive ultrasound guided protocol, we have had no serious cases of morbidity or mortality after liver biopsy.

Audit of the safety of clinical procedures-for example, liver biopsies, is a topical issue. A recent survey of trainees in gastroenterology shows that three quarters wanted to become proficient in ultrasound techniques. ${ }^{13}$ On seeking advice from the various regulatory training bodies, it has been suggested that liver biopsy should be performed using both techniques to prevent loss of expertise in the blind approach. Yet, is it appropriate to reintroduce the blind

Abbreviations used in this paper: JCHMT, Joint Committee on Higher Medical Training. 
technique, and what would the medicolegal position be if a patient died after a blind biopsy and the use of this technique was justified because it was for training purposes? Should we think the unthinkable: that gastroenterologists/hepatologists no longer perform blind liver biopsies, and thus leave experienced radiologists to perform the biopsy using the real time ultrasound guided technique?

We thank Dr AD Beattie of the Advisory Board of the European Board of Gastroenterology and Dr Charles Swan, Chairman of the Specialist Advisory Committee of the Joint Committee for Higher Medical Training, for advice in preparing this manuscript.

S SHAH

J F MAYBERRY

A C B WICKS

Department of Gastroenterology

Department of Radiology

Y REES

R J PLAYFORD

University Division of Gastroenterology, Leicester General Hospital, Gwendolen Road, Leicester LE5 4PW, UK

Correspondence to: Professor Playford (email: rjp13@le.ac.uk).

1 McGill DB, Rakela J, Zinsmeister AR, et al. A 21-year experience with major haemorrhage after percutaneous liver biopsy. Gastroenterology 1990;99:1396-400.
2 Piccinino F, Sagnelli E, Pasquale G, et al. Complications following percutaneous liver biopsy. A multicentre retrospective study on 68276 biopsies. $\mathcal{F}$ Hepatology 1986;2:165-73.

3 Sherlock S. Aspiration liver biopsy, technique and diagnostic application. Lancet 1945;ii:397-401.

4 Westaby D, Macdougall BRD, Williams R. Liver biopsy as a day case procedure: selection and complications in 200 consecutive patients. BMf 1980;281:1331-2.

5 Buscarini L, Fornari F, Bolondi L, et al. Ultrasound-guided fine needle biopsy of focal liver lesions: techniques, diagnostic accuracy and complications. A retrospective study on 2091 biopsies. F Hepatology 1990; 11:344-8.

6 Lindor KD, Bru C, Jorgensen RA, et al. The role of ultrasonography and automatic-needle biopsy in outpatient percutaneous liver biopsy. Hepatology 1996;23:1079-83.

7 Gilmore IT, Burroughs A, IM Murray-Lyon, et al. Indications, methods, and outcomes of percutaneous liver biopsy in England and Wales: an audit by the British Society of Gastroenterology and the Royal College of Physicians of London. Gut 1995;36:437-41.

8 Chuah SY, Moody GA, Wicks ACB, et al. A nationwide survey of liver biopsy: is there a need to increase resources, manpower and training? Hepatogastroenterology 1994;41:4-8.

9 Joint Committee on Higher Medical Training. Curriculum for higher specialist training in general (internal) medicine, fuly 1988. London: Royal College of Physicians, 1998:12-13.

10 Joint Committee on Higher Medical Training. Curriculum for higher specialist training in gastroenterology, fuly 1998. London: Royal College of Physicians, 1998:5.

11 Training the gastroenterologist of the future: The Gastroenterology Core Curriculum. Gastroenterology 1996;110:1266-300.

12 The Education Board of Faculty of Clinical Radiology. Structured training in clinical radiology. London: Royal College of Radiologists, 1995:6-15.

13 What training do gastroenterologists want in abdominal ultrasound? Results of a national survey of trainees [abstract]. Gut 1998;42:A61. 